\title{
Bilateral pan-lobar pneumonia and multiple left lung abscesses in an 11-year-old Nigerian female
}

\section{Case report}

The presenting patient was an 11-year-old Nigerian female, who was admitted on April 13, 2005 to the University of Benin Teaching Hospital (UBTH; Benin - City Edo State, Nigeria).

The patient had previously fallen from a multistorey building with no immediate obvious sequelae. The patient subsequently developed a fever 1 week later, which was continuous, and occurred sporadically for several weeks until presentation at the UBTH. Periods of resolution of the fever usually followed the use of antibiotics, which were prescribed in different hospitals. The patient had her first seizure 2 days after the onset of fever, which was tonic-clonic in nature. Two weeks after the fall she started coughing. It was initially dry, but later became productive of mucus. While on admission, she also experienced post-tussive vomiting of a volume of greenish mucus of $\sim 10 \mathrm{~mL}$.

On examination, the patient was cachectic, illlooking, pale, icteric, with matted tender lymphadenopathy, digital clubbing, dyspnoea and tachypnoea of 56 breaths per min. Her trachea was deviated to the right. Left chest expansion was diminished, with decreased tactile vocal fremitus in the left upper and middle lobes. Vocal fremitus was absent in the left lower zones. There were dull percussion notes in the left upper and middle lobes, and a stony dull note in the lower lobe. A hepatomegaly of $5 \mathrm{~cm}$ below the costal margin was also observed.
HIV infection was ruled out, and an initial diagnosis of pulmonary tuberculosis was made with left lung collapse, consolidation and pleural effusion.

\section{Investigations}

The results of various investigations were as follows: packed cell volume 21\%; white blood cell count 29,400 cells per $\mu$;; neutrophils $78 \%$; lymphocytes 18\%; monocytes 4\%; neutrophils showed moderate left shift with toxic granulations; and erythrocyte sedimentation rate 132 $\mathrm{mm}$ per hour. Sputum microscopy, culture and sensitivity yielded Gram-positive cocci and pus cells, and a Mantoux test result was negative. Retroviral screening also proved negative. Chest radiography showed a dense radio-opaque foreign body in the left lower major bronchus, with collapse and consolidation of the left lung.

\section{M.A. Nzegwu \\ J.U. Aligbe \\ G.I. Eze \\ I.V. Ugwu}

Dept of Morbid Anatomy, University of Benin Teaching Hospital, Benin - City Edo State, Nigeria.

\section{Correspondence:}

M.A. Nzegwu

Consultant Histopathologist

No. 2 Emmanuel Church Road

P.M. B1700 Onitsha

Anambra State

Nigeria

E-mail: martin_nze@yahoo.com

\section{Task 1 \\ What would be your provisional diagnosis at this stage?}




\section{Answer 1 \\ The new working diagnosis was left airway obstruction secondary to foreign body impaction with distal multiple abscesses.}

Further investigation to find and surgically remove the foreign body was planned, but the patient began to experience choking spells, which resulted in the aspiration of bilious fluids and death on the 11 th day of admission.

\section{Autopsy findings}

With regard to the respiratory system, the trachea and left main bronchus were patent, but the right main bronchus was blocked by a foreign body in the proximal third of the length (figure 1). The lungs grossly appeared symmetrical, but were enlarged with prominent rib markings. The left lung was $550 \mathrm{~g}$ in weight and the right $600 \mathrm{~g}$ (normal for age: $400-420 \mathrm{~g}$ ). There was a large hilar lymph node measuring $6 \times 6 \times 5 \mathrm{~cm}$. Both lungs felt solid to touch. The surface was cut and the findings are shown in figure 2 .

In terms of the cardiovascular system, the heart was mildly enlarged and weighed $220 \mathrm{~g}$ (normal for age: $150 \mathrm{~g}$ ). The right side was grossly dilated, with a right atrioventricular valve circumference of $11 \mathrm{~cm}$ (normal for age: 7.5-8 $\mathrm{cm})$. All other parameters were normal.

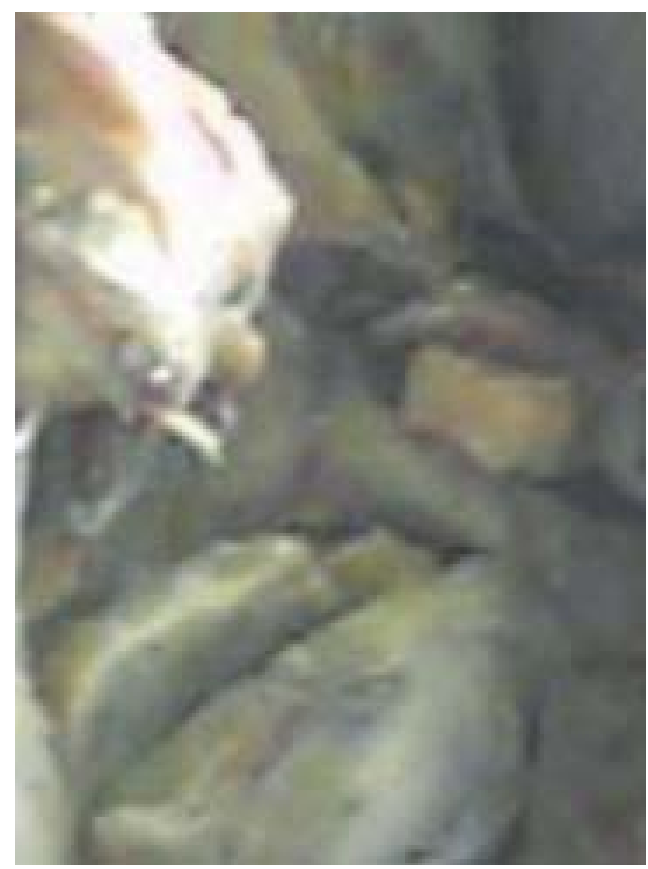

Figure 1

A foreign body impacted in the right main bronchus (posterior view, close up of carina).

\section{Task 2}

Can you identify the foreign body?

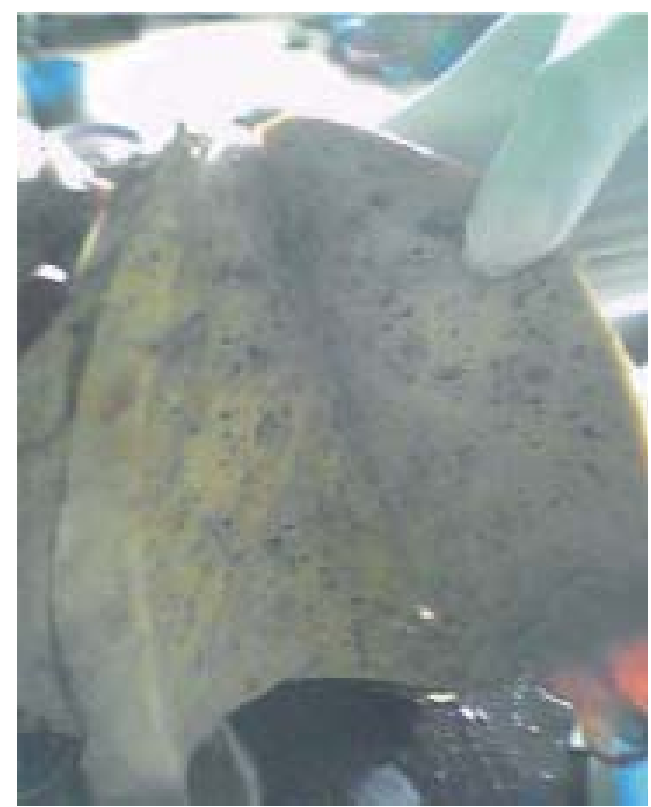

Figure 2

The cut surface of the left lung.

\section{Task 3}

What does the cut lung show?
Task 4 What would you determine as the cause of death? 


\section{Answer 2}

The foreign body was found to be a tooth.

\section{Answer 3}

The cut surface showed a pan lobar consolidation in the whole of the right lung, also affecting the left lung, with multiple small abscesses spread almost evenly in all the lobes.

\section{Answer 4}

Foreign body (tooth) aspiration with left and right pan-lobar pneumonia, and multiple left lung abscesses, in addition to asphyxia and acute cor pulmonale.

\section{Discussion}

Foreign body aspiration can be life threatening [1]. Aspirated solid or semisolid objects can lodge in the larynx or trachea. If the object is large enough to cause obstruction of the airway, asphyxia may rapidly cause death. Lesser degrees of obstruction or passage of the obstructive object beyond the carina can result in less severe signs and symptoms [2]; however, chronic debilitating symptoms with recurrent infection may occur if the foreign body is not removed in good time.

The actual aspiration event can usually be identified, although it is often not immediately appreciated. The aspirated object might even escape detection. Most often, the aspirated object is food [3], but a broad spectrum of aspirated items has been documented over the years. Commonly retrieved objects include seeds, nuts, bone fragments, nails, small toys, coins, pins, medical instrument fragments, and dental appliances and teeth [4]. Geographical differences in the spectrum of objects commonly found in a particular environment, and variations in dietary and eating habits affect the relative frequency with which various objects are aspirated [5].

Here, a case of an 11-year-old Nigerian female is reported. The young female aspirated a tooth as a foreign body. The tooth impacted on the right main bronchus, eventually causing a bilateral pan lobar pneumonia. Multiple left lung abscesses developed due to possible aspiration of infected mucus into the left main bronchus, and death from asphyxia and respiratory failure ensued.

Since the angles made by the mainstream bronchi with the trachea are identical until the age of 15 years, foreign bodies are found with equal frequency in this area in this age group [5]. With normal growth and development, the adult right and left main stream bronchi diverge from the trachea at very different angles, with the right main stem bronchus being more acute and, therefore, developing a relatively straight path from larynx to bronchus. Therefore, in adulthood, objects that descend beyond the trachea are more often found in the right endobronchial tree than in the left.

Cough, dyspnoea, cyanosis and even asphyxia ensued with time in this case presentation. Each human tooth is laden with mouth floral organisms, which probably set up an inflammatory process, with oedema, ulceration, cellular infiltration and granulation tissue formation, further worsening the already established obstruction. The airway is also more likely to bleed with manipulation in this situation, the object then becomes more likely to be obscured and more difficult to dislodge. Distal to the obstruction, air-trapping may occur, leading to local emphysema, atelectasis, hypoxic vasoconstriction, post-obstructive pneumonia or abscesses, and suppurative pneumonia with bronchiectasis [6].

Many aspirated foreign bodies are unexpectedly discovered, go undetected or are misdiagnosed. All three happened in this case. The tooth in question was a premolar whose extraction and aspiration were occult, both probably occurring about the time of the fall. This went undetected for a 2-month period and was only discovered on chest radiography.

\section{References}

1. Limper AH, Prakash UB. Tracheobronchial foreign bodies in adults. Ann Intern Med 1990; 112: 604-609.

2. Chen $C H$, Lai CL, Tsai $\pi$, et al. Foreign body aspiration into the lower airway in Chinese adults. Chest 1997; 112: 129-133.

3. Irwin RS, Ashba JK, Braman SS. Food asphyxiation in hospitalized patients. JAMA 1977; 237: 2744-2745.

4. Fieselmann JF, Zavala DC, Keim LW. Removal of foreign bodies (two teeth) by fibre-optic bronchoscopy. Chest 1977; 72: 241-243.

5. Cleveland RH. Symmetry of bronchial angles in children. Radiology 1979; 133: 89-93.

6. Fraser RG, Pare JA, Pare PD. Pulmonary disease caused by aspiration of solids and liquids. In: Fraser RG, Pare JAP, eds. Diagnosis of Diseases of the Chest. 3rd Edn. UK, WB Saunders Co, 1990; pp. 2383-2416. 